\title{
Dipole matrix element approach vs. Peierls approximation for optical conductivity
}

\author{
P. Wissgott ${ }^{1}$, J. Kuneš ${ }^{2}$, A. Toschi ${ }^{1}$ and K. Held ${ }^{1}$ \\ 1 Institute for Solid State Physics, Vienna University of Technology, 1040 Vienna, Austria \\ 2 Institute of Physics, Academy of Sciences of the Czech republic, \\ Cukrovarnická 10, Praha 6, 162 53, Czech Republic
}

(AUGUST 23, 2021)

\begin{abstract}
We develop a computational approach for calculating the optical conductivity in the augmented plane wave basis set of Wien $2 \mathrm{~K}$ and apply it for thoroughly comparing the full dipole matrix element calculation and the Peierls approximation. The results for $\mathrm{SrVO}_{3}$ and $\mathrm{V}_{2} \mathrm{O}_{3}$ show that the Peierls approximation, which is commonly used in model calculations, works well for optical transitions between the $d$ orbitals. In a typical transition metal oxide, these transitions are solely responsible for the optical conductivity at low frequencies. The Peierls approximation does not work, on the other hand, for optical transitions between $p$ - and $d$-orbitals which usually became important at frequencies of a few eVs.

PACS numbers: 71.27.+a,71.1.Fd
\end{abstract}

Much of our knowledge about solid state systems comes from their response to small electro-magnetic perturbations. A broad range of techniques has been developed to probe the nature of ground states in elastic scattering experiments and the excitations in inelastic scattering or absorption experiments. It is usually a combination of several experimental techniques as well as theoretical calculations which allow us to draw a complete picture of a given material. Among those, the optical spectroscopy plays an important role ${ }^{1}$, complementing the photoemission spectroscopy (PES) which is easier to access and interpret in most theories. Probing the particle-hole excitations averaged over the Brillouin zone, the optical spectra contain a different and less detailed information about the system than angle-resolved photoemission spectra. The main asset of the optical spectroscopy, however, is its robustness: Unlike PES, it does not suffer from surface effects. Moreover, unlike transport measurements, the optical conductivity is not critically affected by impurities or disorder: Optical transitions cannot simply disappear, but can only be shifted to different energies, which is expressed by the sum-rule for optical conductivity ${ }^{2}$.

Calculations of optical spectra from first principles are well established within the effective non-interacting electron theories ${ }^{\underline{3}}$ for weakly correlated materials such as the local density approximation (LDA $)^{4}$ to the density functional theory. The many-body perturbation theory on the GW level ${ }^{5}$ and its two-particle extensions using the Bethe-Salpeter equation ${ }^{6}$ have been successful in describing the excitonic physics in semiconductors. The situation is different in the field of strongly correlated electron systems. Although the optical measurement on these materials proved very useful for investigation of metal-insulator transitions or mass renormalization effects, material specific theoretical investigations are rather rare ${ }^{7-12}$. This is perhaps not surprising given the fact that calculation of one-particle spectra is already a formidable challenge.

In the past decade the dynamical mean-field theory
$(\mathrm{DMFT}) \underline{13} \underline{15}$ combined with first-principles bandstructures $(\mathrm{LDA}+\mathrm{DMFT})^{16} \underline{17}$ showed considerable power to describe correlated materials. This theory allows for an accurate description of the local (intra-atomic) dynamics, while the inter-atomic effects are treated on the static mean-field level. Importantly, DMFT is not restricted to a particular energy scale and thus allows for the simultaneous description of quasiparticles on $\mathrm{meV}$ scale and atomic excitations on the $\mathrm{eV}$ scale, which is crucial to capture the spectral weight transfers in the optical spectra. First optical calculations with DMFT were performed for the single-band Hubbard model. It was shown that the local approximation of DMFT leads to vanishing of the vertex corrections to the optical conductivity $\underline{18-21}$. This means that the electron and hole created in the process of optical excitation behave independently and thus the Green's function of the electron-hole pair is a product of two one-particle Green's functions. This is not necessarily true in multi-band models, except for the case of degenerate bands. However, the dipole selection rules at optical frequencies typically forbid creation of an electron-hole pair on the same atom and thus the vertex corrections may be neglected also in this case, an approximation we also adopt throughout this work. Note that for inelastic x-ray scattering experiments 'optical' transitions with finite momentum transfer allow formation of strongly bound local electron-hole pairs, excitons. The vertex corrections in this case are substantial. A typical example are the crystal-field $d$ - $d$ excitations deep in the optical gap of transition metal oxides; see Ref. ${ }^{22}$ for a $\mathrm{V}_{2} \mathrm{O}_{3}$ calculation.

While the formal framework for calculating the optical conductivity within the above approximations is well established, the numerical implementation poses several challenges: i) $\mathbf{k}$-space integration, ii) determination of the optical transition amplitudes and inclusion of states in a broad energy window, iii) evaluation of optical spectra for real frequencies, which is an additional problem arising for particular numerical techniques, such as quantum Monte-Carlo simulations, used to solve the DMFT equa- 
tions. Different strategies for dealing with these issues are possible. In this article, we present an implementation based on the Wannier functions formalism and a direct calculation of the transition amplitudes from the one-particle wave functions. We compare our results to the so-called Peierls approximation, which relies on the k-derivatives of the effective low-energy Hamiltonian of the systems considered and discuss their relationship. We analyze specifically two well-known correlated oxides, $\mathrm{SrVO}_{3}$ and $\mathrm{V}_{2} \mathrm{O}_{3}$, as archetypes, and compare our results to the available experimental data.

The outline of the paper is as follows: In Section [ we give details on the LDA+DMFT calculation. In Section IIA the technical details of the dipole matrix element caculation are discussed. Section IIB discussed the relationship to the Peierls approximation, which is popular for lattice models. Sections III A and III B present the results for $\mathrm{SrVO}_{3}$ and $\mathrm{V}_{2} \mathrm{O}_{3}$, respectively. Finally, Section IV summarizes the main findings.

\section{LDA+DMFT WITH WANNIER ORBITALS}

The DMFT equations are naturally formulated in terms of fermionic creation and annihilation operators on a lattice, a formulation which assumes an underlying set of localized orthogonal orbitals. Our starting point are the LDA Bloch states $\psi_{n \mathbf{k}}$ and corresponding bandenergies $\varepsilon_{n k}$ calculated with the full-potential linear augmented plane waves (LAPW) program Wien $2 \mathrm{k}: 23,24$ Depending on the specific material considered, we choose an energy window defined by the lower and upper band indices $n_{\min } \leq n \leq n_{\max }$ and transform the states $\psi_{n k}$ from this window to real-space Wannier orbitals $\frac{25}{}$ localized around lattice sites $\mathbf{R}$ :

$$
\left|w_{m \mathbf{R}}\right\rangle=\frac{1}{N_{\mathbf{k}}} \sum_{n \mathbf{k}} e^{i \mathbf{k R}} U_{m n}(\mathbf{k})\left|\psi_{n \mathbf{k}}\right\rangle
$$

where $U(\mathbf{k})$ are unitary matrices defined throughout the Brillouin zone and $N_{\mathbf{k}}$ is the number of k-points. Using wien 2 wannier ${ }^{26}$ and wannier $90^{28}$ the matrices $U(\mathbf{k})$ that lead to maximally localized Wannier functions are found. Construction of the single-particle part of the effective Hamiltonian is completed by rotation of the LDA Hamiltonian into the Wannier basis

$$
H_{m m^{\prime}}^{W}(\mathbf{k})=\sum_{n} U_{m n}^{+}(\mathbf{k}) \varepsilon_{n \mathbf{k}} U_{m n^{\prime}}(\mathbf{k}) .
$$

Finally the on-site interaction is added to the Hamiltonian. Another important input data required for the DMFT calculation, are the local Coulomb repulsion parameters which define the term to be added to $H_{m m^{\prime}}^{W}(\mathbf{k})$ in the Wannier basis: the intra-orbital local repulsion $U$, inter-orbit local interaction $V$ and the exchange parameter $J$. In principle, this input should be computed from the underlying LDA data, with constrained LDA 17 or constrained random phase approximation ${ }^{31}$. However, since identifying $U, V$, and $J$ for $\mathrm{SrVO}_{3}$ or $\mathrm{V}_{2} \mathrm{O}_{3}$ is not the aim of this work, we adapted these values from the literature $33-35,95$. In the case $\mathrm{V}_{2} \mathrm{O}_{3}$ we have chosen a slightly lower value of $U$ than in Refs $\underline{\underline{33}} \underline{\underline{35}}$, which ensures the best agreement with $\mathrm{XAS}^{36}$ and optical experiments ${ }^{37}$, according to considerations reported in

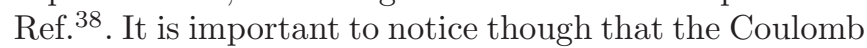
parameter as well as the DMFT itself in general depend on the chosen basis set of Wannier function, which especially becomes important if the choice of Wannier orbitals is not as straightforward as in the case of $\mathrm{SrVO}_{3}$ or $\mathrm{V}_{2} \mathrm{O}_{3}$ below. In both cases the actual DMFT calculation has been done in the $t_{2 g}$ subspace. For the optical conductivity, the DMFT $t_{2 g}$ Green function was then supplemented with the LDA Green function for the other orbitals.

Once the effective LDA Hamiltonian is set up, the DMFT equations are solved numerically using quantum Monte Carlo simulations ${ }^{30}$ with an imaginary time discretization of $\Delta \tau=0.1 \mathrm{eV}^{-1}$ for $\mathrm{SrVO}_{3}$ and $\Delta \tau=0.125$ $\mathrm{eV}^{-1}$ for $\mathrm{V}_{2} \mathrm{O}_{3}$, respectively, to obtain the one-particle self-energy which serves as the many-body input for evaluation of the optical conductivity. Similar LDA+DMFT approaches based on augemted plane waves can be found in Ref. $27,29,32$.

From the LDA+DMFT self energy $\Sigma\left(i \omega_{m}\right)$ for a temperature $T$ at the Matsubara frequencies $\omega_{m}=\pi(2 m+$ 1) $T$, we obtain $\Sigma$ on real frequencies via the procedure described in Ref $\frac{39}{6}$ : Starting from the imaginary-time Green's function $G(\tau)$ measured in Monte-Carlo, the kintegrated spectrum $A(\omega)$ is calculated by the maximum entropy method (MEM, see Ref $\stackrel{40}{\underline{40}}$ ). Afterwards, the local Green's function $G_{M E M}(\omega)$ for real frequencies is found by applying Kramers-Kronig relations. Finally, we fit $\Sigma(\omega)$ such that the Green's function obtained by direct ksummation, i.e. $G(\omega)=1 / N_{\mathbf{k}} \sum_{\mathbf{k}}\left[\omega-H_{W}(\mathbf{k})-\Sigma(\omega)\right]^{-1}$, matches the one from the maximum entropy method.

\section{LINEAR RESPONSE FOR THE OPTICAL CONDUCTIVITY}

\section{A. Dipole matrix element approach}

The regular part of the optical conductivity is obtained via the standard Kubo's formula in linear response ${ }^{2}$

$$
\sigma_{\alpha \beta}(\omega)=\lim _{\mathbf{q} \rightarrow 0} \operatorname{Re}\left(\frac{1}{\omega V} \int d t e^{i \omega t}\left\langle\left[j_{\alpha}(\mathbf{q}, t), j_{\beta}(-\mathbf{q}, 0)\right]\right\rangle\right)
$$

where $V$ is the unit cell volume, $j_{\alpha}(\mathbf{q}, t)$ is the $\mathbf{q}$ momentum paramagnetic current in $\alpha$ direction, $\hbar=1$ and $q \rightarrow 0$ is the dipole approximation. Expressing Eq. (3) via the Lindhardt bubble and Matsubara formalism (thus omitting vertex corrections, consistently with the discussion above), we include internal degrees of freedom describing optical excitations ${ }^{2}$ : initial (final) frequency $\omega,(\omega+\Omega)$, reciprocal vector $\mathbf{k}$ and $N=n_{\max }-$ 
$n_{\text {min }}$ orbital degrees of freedom participating in optical transitions. Altogether, we obtain the following expression for the real part of the optical conductivity

$$
\begin{aligned}
\sigma_{\alpha \beta}(\omega)= & \frac{2 \pi}{V} \sum_{\mathbf{k}} \int \mathrm{d} \omega^{\prime} \frac{f\left(\omega^{\prime}\right)-f\left(\omega^{\prime}+\omega\right)}{\omega} \\
& \times \operatorname{Tr}\left[A\left(\mathbf{k}, \omega^{\prime}\right) v_{W}^{\alpha}(\mathbf{k}) A\left(\mathbf{k}, \omega^{\prime}+\omega\right) v_{W}^{\beta}(\mathbf{k})\right]
\end{aligned}
$$

where $f$ is the Fermi function, $v_{W}^{\alpha}(\mathbf{k})=U(\mathbf{k}) v^{\alpha}(\mathbf{k}) U^{+}(\mathbf{k})$ are rotated matrix elements of the momentum operator $v_{n m}^{\alpha}(\mathbf{k})=-i\left\langle\psi_{n \mathbf{k}}\left|\nabla_{\alpha}\right| \psi_{m \mathbf{k}}\right\rangle / m_{e}, 1 \leq, n, m \leq N$, the elementary charge $e=1$, and $A(\mathbf{k}, \omega)=-\operatorname{Im} G(\mathbf{k}, \omega) / \pi$ is the generalized spectral function with the Greens function

$$
G(\mathbf{k}, \omega)=\left[(\omega+\mu) \mathbb{1}-H_{W}(\mathbf{k})-\Sigma(\omega)\right]^{-1} .
$$

Here, $\mu$ denotes the chemical potential, $H_{W}(\mathbf{k}) \in \mathbb{C}^{N \times N}$ the (non-interacting) Hamiltonian in the Wannier orbital basis and $\Sigma(\omega) \in \mathbb{C}^{N \times N}$ the self energy from the LDA+DMFT calculation.

For an efficient and accurate k-quadrature of Eq. (4), we use a tetrahedral-mesh integration. To resolve regions in k-space with larger integration error we adaptively refine the tetrahedra in these domains. Furthermore, the symmetry operations of the unit cell are applied such that the integrand of Eq (4) has to be evaluated only at kpoints within the reduced wedge of the Brillouin zone. The computation of the momentum matrix elements

$$
v_{n m}(\mathbf{k})=-i \frac{\left\langle\psi_{n \mathbf{k}}|\nabla| \psi_{m \mathbf{k}}\right\rangle}{m_{e}}
$$

which are in the following also denoted as dipole matrix requires their evaluation in term of the underlying LAPW basis set프. It is thus (to our knowledge for the first time) possible to combine a full potential LAPW dipole matrix with Wannier-functions-based DMFT algorithms for the computation of transport and optical properties (for different approaches see e.g. Refs $\stackrel{42,43}{\underline{4} \text { ). }}$. Note that the surveyed workflow is not limited to the use of a DMFT self energy $\Sigma(\omega)$, but can be easily generalized for other, even $\mathbf{k}$-dependent self energies $\Sigma(\mathbf{k}, \omega)$. In such cases, however, the inclusion of vertex corrections to the bubble term becomes usually necessary $\underline{\underline{44}}$.

In addition to transitions within Hilbert space of the low energy model, the present approach also allows inclusion of higher energy bands. This can be achieved by enlarging the transformation matrices $U(\mathbf{k})$ and, consequently, the Hamiltonian $H_{W}(\mathbf{k})$

$$
\begin{aligned}
\mathcal{U}(\mathbf{k}) & =\left[\begin{array}{ccc}
\mathbb{1} & 0 & 0 \\
0 & U(\mathbf{k}) & 0 \\
0 & 0 & \mathbb{1}
\end{array}\right], \\
\mathcal{H}(\mathbf{k})= & {\left[\begin{array}{ccc}
E^{(1)}(\mathbf{k}) & 0 & 0 \\
0 & H_{W}(\mathbf{k}) & 0 \\
0 & 0 & E^{(2)}(\mathbf{k})
\end{array}\right] }
\end{aligned}
$$

with diagonal $E_{n}^{(1)}(\mathbf{k}), E_{n}^{(2)}(\mathbf{k})=\varepsilon_{n \mathbf{k}}$ for $n<n_{\min }(n>$ $\left.n_{\max }\right)$. Note that though the $\mathcal{U}$ and $\mathcal{H}_{W}$ are blockdiagonal, the corresponding dipole matrix $\mathcal{V}(\mathbf{k})=$ $\mathcal{U}(\mathbf{k}) v^{\alpha}(\mathbf{k}) \mathcal{U}^{+}(\mathbf{k})$ is not. Inserting $\mathcal{U}, \mathcal{H}, \mathcal{V}$ into Eq. (4) and (5), we thus also take transitions between the Wannier orbitals and Bloch states outside of the low energy model into account.

\section{B. Peierls approximation}

For many-body calculations of lattice models, it is common practice to determine the optical conductivity by the Peierls approximation $(\mathrm{PA})^{45}$. The PA approximates the group velocities directly from the hopping elements and, for non-Bravais lattices, from the atomic positions in the unit cell9,10,12. If one wants to go beyond the PA, however, one needs to know the underlying continuum description for calculating the dipole matrix elements. The idea of the PA is a gauge transformation of the electro-magnetic potential $\mathbf{A}$ which disregards the inner orbital structure (an orbital will get a different gauge factor at different positions) and assumes a single gauge factor which only depends on the lattice site. This is reflected in a modified hopping amplitude $t_{\mathbf{R} m ; \mathbf{R}^{\prime} n} \rightarrow t_{R m ; R^{\prime} n} \exp \left[i \mathbf{A}\left(\mathbf{R}-\mathbf{R}^{\prime}\right) / c\right]^{12,21}$ between sites $\mathbf{R}$ and $\mathbf{R}^{\prime}$.

In the following we discuss the corrections to the PA, emerging from the exact continuum description in the Wannier orbitals basis, cf. Ref. $\underline{12}$. Using the operator identity $-\frac{1}{m} \nabla=\left[H_{0}, \mathbf{r}\right]$, where $H_{0}$ is the one-particle part of the Hamiltonian we can write the momentum matrix element as

$$
\begin{aligned}
& \frac{-1}{m_{e}}\left\langle w_{m \mathbf{k}}|i \nabla| w_{m^{\prime} \mathbf{k}}\right\rangle=\frac{i}{N} \sum_{\mathbf{R}, \mathbf{R}^{\prime}} e^{i \mathbf{k}\left(\mathbf{R}^{\prime}-\mathbf{R}\right)}\left\langle w_{m \mathbf{R}}\left|\left[H_{0}, \mathbf{r}\right]\right| w_{m^{\prime} \mathbf{R}^{\prime}}\right\rangle \\
& =\frac{i}{N} \sum_{\mathbf{R}, \mathbf{R}^{\prime}} e^{i \mathbf{k}\left(\mathbf{R}^{\prime}-\mathbf{R}\right)}\left[\left(\mathbf{R}^{\prime}-\mathbf{R}\right)\left\langle w_{m \mathbf{R}}\left|H_{0}\right| w_{m^{\prime} \mathbf{R}^{\prime}}\right\rangle\right. \\
& \left.+\left\langle w_{m \mathbf{R}}\left|H_{0}\left(\mathbf{r}-\mathbf{R}^{\prime}\right)\right| w_{m^{\prime} \mathbf{R}^{\prime}}\right\rangle-\left\langle w_{m \mathbf{R}}\left|(\mathbf{r}-\mathbf{R}) H_{0}\right| w_{m^{\prime} \mathbf{R}^{\prime}}\right\rangle\right] .
\end{aligned}
$$

The first term equals $\nabla_{\mathbf{k}} H(k)$, the PA, and can be obtained without explicit knowledge of the orbital, e.g. from an empirical tight-binding Hamiltonian. The remaining two terms can be further analyzed by noting that the Wannier functions form a complete eigenbasis of $H_{0}$. Hence we have

$$
-\frac{1}{m_{e}}\left\langle w_{m \mathbf{k}}|i \nabla| w_{m^{\prime} \mathbf{k}}\right\rangle=\nabla_{\mathbf{k}} H_{m m^{\prime}}(\mathbf{k})+\mathcal{C}_{m m^{\prime}}(\mathbf{k}),
$$


where

$$
\begin{aligned}
& \mathcal{C}_{m m^{\prime}}(\mathbf{k})=\frac{i}{N} \sum_{\mathbf{R}, \mathbf{R}^{\prime}} e^{i \mathbf{k}\left(\mathbf{R}^{\prime}-\mathbf{R}\right)} \times \\
\sum_{\mathbf{L}, p} & {\left[\left\langle w_{m \mathbf{R}}\left|H_{0}\right| w_{p \mathbf{L}}\right\rangle\left\langle w_{p \mathbf{L}}\left|\mathbf{r}-\mathbf{R}^{\prime}\right| w_{m^{\prime} \mathbf{R}^{\prime}}\right\rangle\right.} \\
& \left.-\left\langle w_{m \mathbf{R}}|\mathbf{r}-\mathbf{R}| w_{p \mathbf{L}}\right\rangle\left\langle w_{p \mathbf{L}}\left|H_{0}\right| w_{m^{\prime} \mathbf{R}^{\prime}}\right\rangle\right] .
\end{aligned}
$$

Let us first discuss the corrections for a single atom in the unit cell. These can be classified as follows

(i) Intra-atomic dipole transitions: Terms in Eqs . (9) and (11) with $\mathbf{R}=\mathbf{R}^{\prime}$ yield together $\left\langle w_{m \mathbf{R}}\left|\left[H_{0}, \mathbf{r}\right]\right| w_{m^{\prime} \mathbf{R}}\right\rangle$, i.e., the atomic-dipole elements with the only difference being that $H_{0}$ is the one-particle Hamiltonian of the solid and not of the atom. These local transitions generally require different angular momenta for $m$ and $m^{\prime}$ orbitals and are hence at a higher energy. They cannot be described by the PA which only considers a single gauge factor for the atom or site.

(ii) Dipole transition mediated hopping: For $\mathbf{R}^{\prime}=$ $\mathbf{L} \neq \mathbf{R}$, the first term of Eq. (11) consists of a hopping integral $\left\langle w_{m \mathbf{R}}\left|H_{0}\right| w_{p \mathbf{R}^{\prime}}\right\rangle$ and a local dipole transition $\left\langle w_{p \mathbf{R}^{\prime}}\left|\mathbf{r}-\mathbf{R}^{\prime}\right| w_{m^{\prime} \mathbf{R}^{\prime}}\right\rangle$. This is similar as intraatomic dipole transitions, however now we obtain a $\mathbf{k}$ dependence which was absent for (i). Note, the same is obtained for the second term of Eq. (11) in the case $\mathbf{R}=\mathbf{L} \neq \mathbf{R}^{\prime}$.

(iii) Inter-atomic dipole transitions: For $\mathbf{R}=\mathbf{L} \neq \mathbf{R}^{\prime}$, the first term of Eq.(11) consists of a local Wannier matrix element $\left\langle w_{m \mathbf{R}}\left|\hat{H}_{0}\right| w_{p \mathbf{R}}\right\rangle$ and an inter-atomic dipole transition $\left\langle w_{p \mathbf{R}}\left|\mathbf{r}-\mathbf{R}^{\prime}\right| w_{m^{\prime} \mathbf{R}^{\prime}}\right\rangle$. A similar term with a minus sign is obtained for the second term of Eq. (11) in the case $\mathbf{R}^{\prime}=\mathbf{L} \neq \mathbf{R}$. If the orbitals are locally orthogonal, only the local on-site energies survive, and we only get a contribution if there is a crystal field splitting of the orbitals.

(iv) Further corrections arise if all lattice positions $\mathbf{R}$, $\mathbf{L}$ and $\mathbf{R}^{\prime}$ are different in Eq. (11). In this case we have a combination of an inter-atomic dipole element and a hopping term.

If the orbitals are more localized, i.e., exponentially decaying between the atoms, both the hopping element and the inter-atomic dipole element are affected by this exponential suppression. Hence the terms (iv), which contain two such exponentials, are more strongly suppressed than the hopping amplitude itself (which enters (ii) and (iii) as well as the PA) since it only contains one exponential factor. The leading term in the "localized" limit is (i), which only involves local transitions.

From these general considerations, the PA appears a rather unjustified approximation. In fact, even in the limit of more localized orbitals only the terms (iv) get suppressed. However, in specific cases of interest the PA may be justified. For instance, terms (i)-(iii) become only relevant if the orbitals are (a) affected by a large crystal field splitting or (b) of a different angular momentum, which typically also means large excitation energies.
Hence for transitions below this energy, e.g. the Drude peak, PA is expected to work, at least for sufficiently localized orbitals.

The situation becomes a bit more involved in the case of several atoms in the unit cell. Tomczak and Biermann 9,10 showed that the PA has to be generalized to include the hopping terms between the atoms in the same unit cell, which are absent in $\nabla_{\mathbf{k}} H_{m m^{\prime}}(\mathbf{k})$. However, also in this case, the same correction terms (i)-(iv) as discussed above remain.

\section{RESULTS}

\section{A. $\mathrm{SrVO}_{3}$}

Due to its simple cubic (perovskite) lattice structure and $3 d^{1}$ electronic structure, $\mathrm{SrVO}_{3}$ has been employed as a testbed for $a b$ initio calculations such as LDA+DMFT. There is, on average, a single $d$ electron residing in three degenerate $t_{2 g}$ bands that cross the Fermi energy $E_{F}$. These $t_{2 g}$ bands are well separated by a gap from the oxygen $p$ bands below and the $e_{g}^{\sigma}$ orbitals above. This situation makes the electronic structure of $\mathrm{SrVO}_{3}$ particularly simple.

The photoemission spectra ${ }^{46}-49$ show a well developed lower Hubbard below $E_{F}$ band and a pronounced quasiparticle peak around $E_{F}$; an upper Hubbard band is found, on the other side, in x-ray absorption experiments 48 The quasiparticle peak is renormalized (narrowed) by a factor of about 2 compared to the overall LDA $t_{2 g}$ bandwidth ${ }^{47}$ This is in good agreement with $\mathrm{LDA}+\mathrm{DMFT}$ calculations 47,95 in which the interaction parameters have been determined from constrained LDA calculations ${ }^{50}$. Essentially the same one-particle spectrum has also been obtained in subsequent LDA+DMFT calculations (among others see Refs. 27,32,51-56); and various Wannier function projection schemes have been tested for this prototypical material (among others see Refs $\stackrel{57-61}{-}$ ). $\mathrm{SrVO}_{3}$ is also the materials where kinks in strongly correlated electron systems, abstain from any external bosonic degrees of freedom and anti-ferromagnetic spin-fluctuations have been discovered.51,62 Similar structures can also be identified in angular resolved photoemission spectra. ${ }^{63}$ As the optical conductivity averages (integrates) however over different k-points, such fine structures are hardly discernible in this physical quantity $\underline{64}$ Experimentally, the optical conductivity shows a Drude peak and additional features above $2 \mathrm{eV}$ when transitions between Hubbard and quasiparticle peak become relevant (among others see Refs. $\left.{ }^{65,66}\right)$.

\section{Spectral properties}

The LDA density of states (DOS) for $\mathrm{SrVO}_{3}$ used in our analysis can be found in Fig. 1] (top panel), where 
the three partial DOS contributions $\mathrm{V}-t_{2 g}, \mathrm{~V}-e_{g}$ and O$p$ are highlighted (we use $a=3.84 \AA$ as unit cell lattice parameter for the cubic perovskite). From the LDA data, we obtained three different Wannier projections: First, just the $\mathrm{V}-t_{2 g}$ manifold was mapped onto three Wannier orbitals (in the following abbreviated as $P 1$ ). Second, we also included the two additional bands with predominant $\mathrm{V}-e_{g}$ character and thus describe the full $\mathrm{V}-d$ manifold $(P 2)$. Finally, we also take into account the O- $p$ bands which leads to a basis consisting of 14 Wannier functions $(P 3)$.

In Fig. 1. middle and lower panel, we plot the LDA+DMFT spectra computed with the Wannier basis sets $P 1$ and $P 2$, respectively. The parameters where adapted from Ref $\frac{95}{}$ : local intra-orbital Coulomb repulsion $U=5.05 \mathrm{eV}$, local inter-orbital repulsion $V=3.55$ $\mathrm{eV}$, and local exchange $J=0.75 \mathrm{eV}$. Compared to the LDA DOS, the $t_{2 g}$ partial density of states is renormalized and the formation of lower and upper Hubbard bands can be observed as correlation effects are taken into account within the DMFT framework. In the case of $P 2$, where all $5 \mathrm{~V}$ Wannier orbitals are included in the DMFT, the $e_{g}$ orbitals remain completely unoccupied as in LDA leading to negligible correlation effects in these two orbitals (see lower panel of Fig. (1). We thus restricted the LDA+DMFT analysis for lower temperatures to $P 1$, where only the $t_{2 g}$ orbitals are described within DMFT.

2. Comparison of the dipole matrix elements approach and the Peierls approximation

In Fig. 2, our main results for the optical conductivity of $\mathrm{SrVO}_{3}$ are summarized. We compare four different calculations for the (isotropic) optical conductivity $\sigma$ computed via Eq.(4) with the experimental data from Ref $\frac{65}{}$ : The uppermost panel shows $\sigma$ computed by use of the LDA Greens function (5), where we fixed the broadening by setting $\Sigma=-0.04 i[\mathrm{eV}]$ in Eq.(15), and employed the dipole matrix (6) as group velocities. The second panel of Fig. 2, visualizes the optical conductivity $\sigma$ computed with the same Green function $G(\mathbf{k}, \omega)$, but with the Peierls approximation $\nabla_{\mathbf{k}} H_{W}(\mathbf{k})$ for the group velocities (we are neglecting for this calculation the intraunit-cell contributions as introduced by Tomczak ${ }^{10}$ ). For the lower two panels, we inserted the DMFT self energy into the formula for the Greens function (5). In particular, the third and the fourth panel of Fig. 2 show the LDA+DMFT results for $\sigma$ calculated with the dipole matrix and the Peierls approximation as group velocities, respectively. Note that the effect of taking a different temperature in the experiment $(T=290 \mathrm{~K})$ and in the calculations $(T=460 \mathrm{~K})$ is expected to be limited since in this temperature range $\mathrm{SrVO}_{3}$ does not show a notable change in the electronic structure. The main consequence of lowering the temperature $T=460 \rightarrow 290$ is the decreased electron-electron scattering within the coherent part of the electron spectrum which eventually leads to

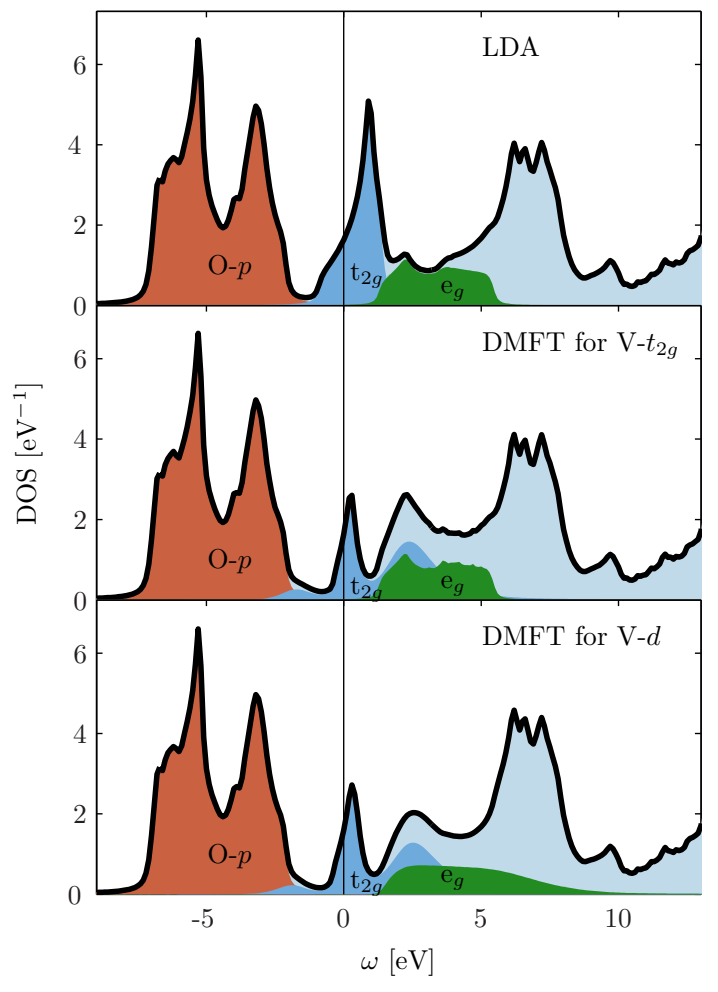

FIG. 1. (Color online) Non-interacting partial density of states of $\mathrm{SrVO}_{3}$ (results abbreviated as LDA) compared to DMFT spectra at $T=1160 K$. Two DMFT basis sets were employed: first, the three orbital $\mathrm{t}_{2 g}$ basis with parameters $(U, J, V)=$ $(5.05,0.75,3.55) \mathrm{eV}$; second, the entire $\mathrm{V} \mathrm{d}$ manifold with the same interaction parameters for all orbitals.

the Drude peak to become more pronounced while the inter-band contributions remain essentially unchanged.

In our analysis of the results, let us start investigating the qualitative effect of correlation on the optical spectra, i.e. comparing the upper two panels of Fig. 2 with the two lower ones. The renormalization of the $t_{2 g}$ manifold surveyed in Fig. 1 1 leads to a smaller Drude weight in the LDA+DMFT panels of Fig. 2 and to a suppression of the prominent peak of $\sigma$ around $3.5 \mathrm{eV}$ predominately stemming from transitions from the occupied O- $p$ manifold to the unoccupied section of the V-t $t_{2 g}$ orbitals. The suppression of these two features is also seen in experiment. Additionally, the DMFT optical spectra in the lower two panels of Fig. 2 show the formation of a small satellite at $\sim 2 \mathrm{eV}$ originating from transitions from the lower Hubbard band of the $t_{2 g}$ orbitals to the unoccupied part of their coherent spectral peaks.

Comparing the dipole matrix approach with the Peierls approximation, i.e. the first with the second and the third with the fourth panel in Fig. 2 indicates both reproduce low energy transitions in a similar way. Since the Drude peak in this material stems from intra-band excitations of the $t_{2 g}$ bands, this implies that the Peierls approximation is sufficient to describe optical transitions in $\mathrm{SrVO}_{3}$ as long as only well localized orbitals are participating. The 


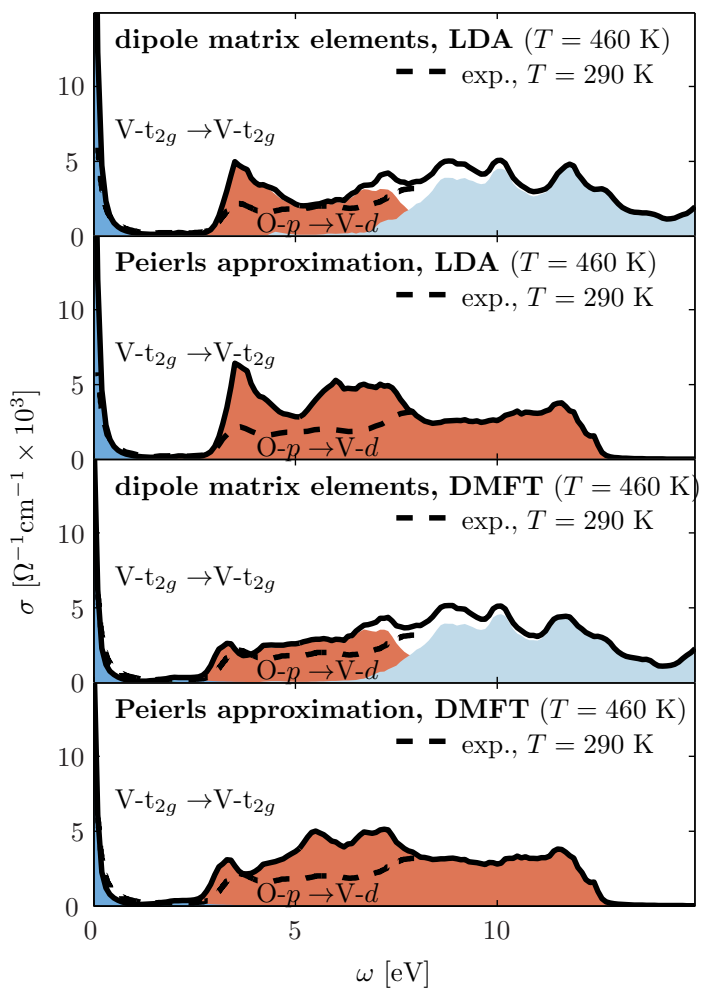

FIG. 2. (Color online) Optical conductivity of $\mathrm{SrVO}_{3}$ calculated with dipole matrix elements and the Peierls approximation, respectively, compared to experiment ${ }^{65}$.

case is different for the $\mathrm{O}-p \rightarrow \mathrm{V}-t_{2 g}$ transitions, where a deviation in the range between $4 \mathrm{eV}$ and $13 \mathrm{eV}$ is clearly visible. Here, the dipole matrix matrix approach appears to be superior and $\sigma$ is much closer to the experimental results than the Peierls approximation, especially for the LDA+DMFT optical spectra (see third and fourth panel of Fig. (2). The reason for this behavior can be understood taking into account the more non-local nature of the O- $p$ orbitals and the deficiency of the Peierls approximation to describe optical transitions therein quantitatively correct.

In addition to deviations compared to the choice of group velocities, both LDA+DMFT results for the optical spectra deviate with experiment around $3.5 \mathrm{eV}$ at the onset of the $\mathrm{O}-p \rightarrow \mathrm{V}-t_{2 g}$ transitions. Since LDA seems to describe this onset more accurately, we think that the reason for this behavior deduces from the fact that, including only the $t_{2 g}$ orbitals in LDA+DMFT, we did not consider a double counting correction shifting the $t_{2 g}$ orbitals relative to the O- $p$ orbitals. A more complete approach would consider the O- $p$ within LDA+DMFT on the level of the Hartree approximation taking into account the double counting corrections more accurately. Then, the change of the $t_{2 g}$ orbitals within LDA+DMFT would eventually shift the $p$ states to lower energies correcting the energy distance between the onset of the O- $p$ manifold to the (now renormalized) peak in the $t_{2 g}$ orbitals back to the LDA level.

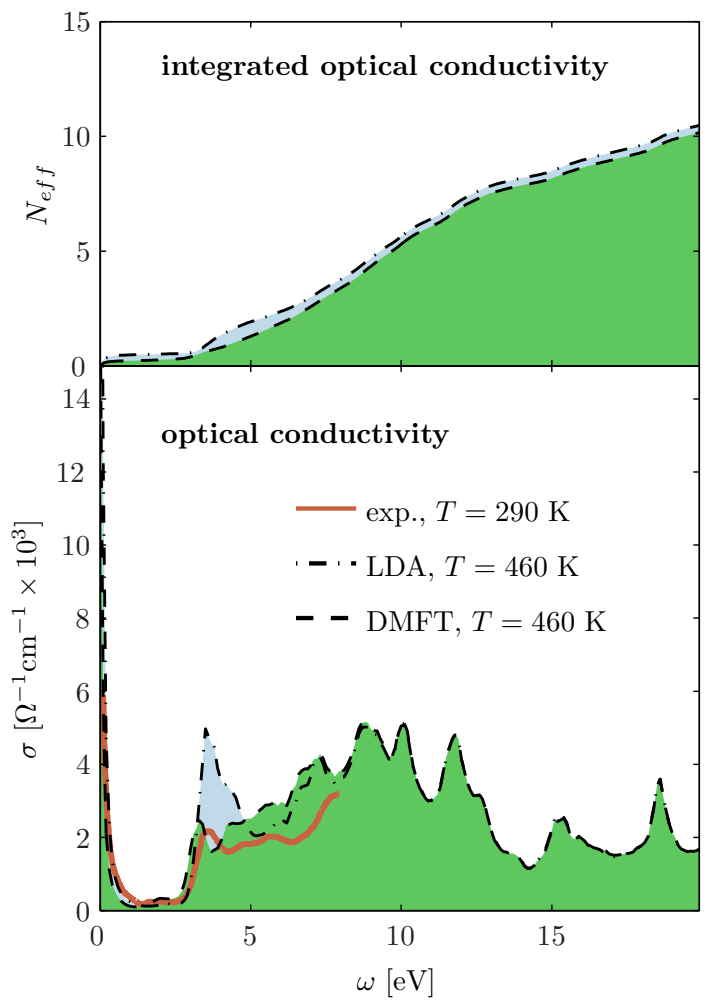

FIG. 3. (Color online) Optical conductivity of $\mathrm{SrVO}_{3}$ comparing LDA, DMFT and experiment. The LDA and DMFT results were computed by the dipole matrix element approach (bottom). The top panel shows the sumrule $N_{\text {eff }}\left(\Omega_{c}\right)$ from Eq. (12) for $\sigma$ of the lower panel (the experimental data are from Ref. ${ }^{65}$.

\section{Sumrule analysis}

An important aspect associated to the theoretical and experimental study of the optical spectroscopy is the analysis of the associated f-sum rule ${ }^{2}$. This is a direct consequence of charge conservation, stating that the integral over all frequencies of the optical conductivity is always proportional to the total electronic density $n_{t o t}=N_{t o t} / V$ of the system

$$
\lim _{\Omega_{c} \rightarrow \infty} \frac{m_{e}}{\pi e^{2}} \int_{0}^{\infty} d \omega \sigma(\omega)=\lim _{\Omega_{c} \rightarrow \infty} \frac{N_{e f f}\left(\Omega_{c}\right)}{V}=\frac{N_{t o t}}{V} .
$$

The importance of the f-sum rule, however, goes well beyond the verification of the charge conservation in LDA or LDA+DMFT calculations of optical spectra. The validation of Eq. (12) in theoretical calculations as well as in experiment represents a rather academic but delicate issue, as it involves very different energy scales (corresponding to optical transitions involving valence and core states). For further details about this issue, we refer the reader to Refs $\underline{68,69}$. 


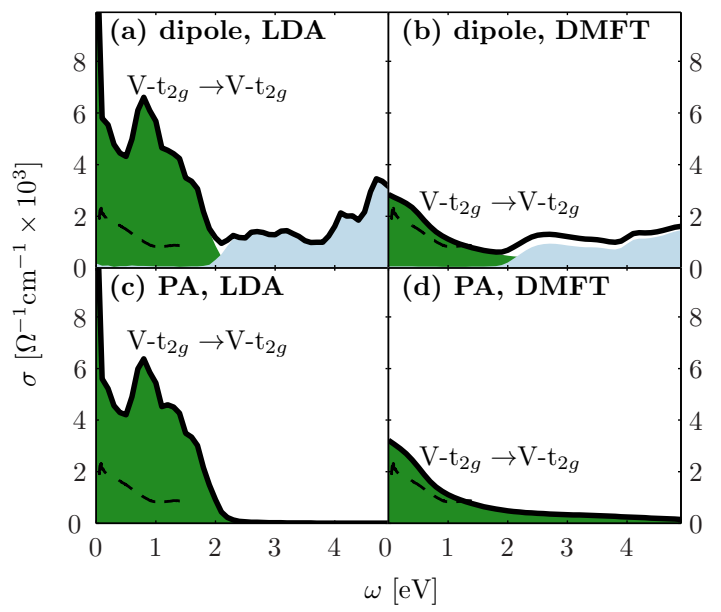

FIG. 4. (Color online) Optical conductivity of $\mathrm{V}_{2} \mathrm{O}_{3}$ ( $\alpha$-phase, metallic) at $T=460 \mathrm{~K}$ calculated with dipole matrix elements [a: LDA, b: DMFT for $\mathrm{V}-\mathrm{t}_{2} g$ with $(\mathrm{U}, \mathrm{J}, \mathrm{V})=(4,0.7,2.6)$ $\mathrm{eV}$ ] and the Peierls approximation [c: LDA, d: DMFT for V$\mathrm{t}_{2} g$ with $\left.(\mathrm{U}, \mathrm{J}, \mathrm{V})=(4,0.7,2.6) \mathrm{eV}\right]$, respectively, compared to experiment (dashed line taken from Ref ${ }^{78}$ ).

More specific information can be extracted by the analysis of so-called partial or restricted optical sum-rules. They correspond to consider just a portion of the frequency integral in Eq.12, a typical case being a finite upper cut-off $\Omega_{c}$, and how this partial integral changes as a function of external parameters (e.g., temperature, magnetic field, etc...). This provides usually very important information about the energy balance associated, e.g,. with a phase transitions, as it has emerged from many experimental ${ }^{70}$ and theoretical analysis $\underline{\underline{ }} \underline{ }$ of integrated optical spectroscopic data of high-temperature superconducting cuprates, and most recently, by analyzing 72 the non-Slater nature of the antiferromagnetic phase in the optical spectra of $\mathrm{LaSrMnO}_{4}{ }^{73}$.

An example for the application of Eq. (12) is reported in the upper panel of Fig. 3, where the growth of $N_{\text {eff }}$ with increasing frequency up to $\Omega_{C}=20 \mathrm{eV}$ is shown for the case of $\mathrm{SrVO}_{3}$ (there are 19 valence electrons included in our calculation). While a detailed analysis of the restricted sum rules for $\mathrm{SrVO}_{3}$ goes beyond the scope of this work (for the analysis of the restricted sum rule in $\mathrm{V}_{2} \mathrm{O}_{3}$, see, e.g., Ref $\stackrel{75}{ }$ ), when comparing the integrated LDA and LDA+DMFT optical spectra of Fig. 3, we can note, for the latter case, a slight decrease of the values of $N_{\text {eff }}$ in the low-frequency region, which reflects, evidently, a correspondent reduction of the electronic mobility due to electronic correlations. At higher frequency, however, the LDA electron density value has been recovered within an accuracy of about $3 \%$.

\section{B. $\mathbf{V}_{2} \mathbf{O}_{3}$}

Vanadium sesquioxide $\mathrm{V}_{2} \mathrm{O}_{3}$ has been subject of a considerable interest in condensed matter physics since the early Seventies (see e.g. Ref $\stackrel{74}{ }$ ), as it represents one of the most evident realization of the Mott-Hubbard metalto-insulator transition (MIT). In fact, $\mathrm{V}_{2} \mathrm{O}_{3}$ can be relatively easily doped with $\mathrm{Cr}$ or $\mathrm{Ti}$, and its phase diagram displays a clear first order transition between a paramagnetic metallic (PM) state (at low concentration of $\mathrm{Cr}$, or for Ti doping) and a paramagnetic insulating (PI) at a higher level of Cr doping. Such a first order MIT, which emerges from a (simultaneous) lower temperature structural and magnetic transition and ends up at higher $T$ s with a second order (critical) endpoint, is completely isostructural: The high- $T$ paramagnetic phases of $\mathrm{Cr}_{x^{-}}$ $\mathrm{V}_{2-x} \mathrm{O}_{3}$ are always associated with a corundum crystal structure.

The experimental evidence of the MIT in $\mathrm{V}_{2} \mathrm{O}_{3}$ has been accumulated, first for static quantities (e.g., the dc resistivity) and -ar a later time- for spectral functions (PES흐, ARPES 37 , XAS 36,77 , etc...). In this paper, however, we focus on infrared-optical spectroscopy $37,75,78$ only, which is a bulk sensitive technique in comparison to photoemission, and -contrary to XAS- includes important information about the itinerant part of the electronic properties of strongly correlated electron systems. In optical spectroscopy measurements at room $T$, the crossing of the MIT upon Cr-doping is clearly reflected in the abrupt disappearance of the (weak) Drude peak in the in-plane ${ }^{79}$ optical conductivity $\sigma(\omega)$ with the opening of a sizable spectral gap. Further important information has been also extracted from the temperature ${ }^{75}$ and pressure ${ }^{37}$ dependence of $\sigma(\omega)$ : The former has provided a clear indication of a strong interplay between small changes of the lattice parameters and electronic properties, while the latter (together with XAS measurements of the V K-pre-edge) has proven the inconsistency of the long-standing assumption of equivalence of doping-level and applied pressure in the phase diagram of $\mathrm{V}_{2} \mathrm{O}_{3}$. Also to be mentioned are very recent optical measurements 37 performed in the most "intriguing" region of the phasediagram, i.e., right across the MIT first order transition line: The combined analysis of optical data and photoemission on a microscopic scale has demonstrated the formation of insulating islands embedded in the PM phase in the metallic side of the MIT. The formation of such islands, growing in size when the transition is approached, can be put -to some exert- in analogy with the nucleation processes due to impurities in a standard liquid-gas transition: In the case of $\mathrm{V}_{2} \mathrm{O}_{3}$ the impurity would be likely provided by the lattice distortions ${ }^{80}$ due to the Cr- substitutions.

From the theoretical point of view, the problem to be analyzed consists of a systems with two electrons in the three $3 d-t_{2 g}$ (i.e., correlated) bands of the $V$ atom at the Fermi level. The $t_{2 g}$ basis further splits in one $a_{1 g}$ and two $e_{g \pi}$ local orbitals (separated by $0.2-0.3 \mathrm{eV}$ ) because of a slight trigonal distortion of the material (see, e.g., LDA calculations with $N$ th order muffin-tin

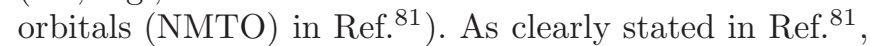
the interplay between strong electronic correlation and 
multi-orbital physics is expected to be the crucial ingredient of the physics underlying the Mott MIT in in $\mathrm{V}_{2} \mathrm{O}_{3}$. In fact, the properties of the MIT in the Cr-doped $\mathrm{V}_{2} \mathrm{O}_{3}$ have been calculated (in some case even preceding the experimental results) by means of LDA+DMFT in Refs $\frac{34.35}{4}$, and, later, by including the orbital hybridization in Ref 33 .

Beside the success in describing photoemission data, LDA+DMFT can be also used to analyze optical spectra. While -at the DMFT level- the numerical effort for computing the optical conductivity $\sigma(\omega)$ is comparable to that for computing spectral functions, as vertex corrections can be usually neglected $\frac{18}{}$, rough approximations have been always done in evaluating the optical dipole matrix elements in the localized (NMTO, Wannier, etc..) orbital basis. In particular, in the first LDA+DMFT calculations of $\sigma(\omega)$ for $\mathrm{V}_{2} \mathrm{O}_{3} \frac{75}{1}$ the dipole matrix elements were simply replaced by 1 , while in later works 9 the dipole matrix elements have been evaluated in the Peierls approximation, including the effects of multiple atoms in the unit cell when necessary 10.12 .

Our results for $\mathrm{V}_{2} \mathrm{O}_{3}$ are summarized in Fig. 4, where we show in the first row LDA (left) and LDA+DMFT (right) calculations for the optical conductivity obtained by using the optical matrix elements, while in the second row the corresponding calculations made with the PA are discussed (we use $a=4.95 \AA, c=14 \AA$ as lattice parameters, see Ref. .11 and references therein). In all cases, we also directly compare our theoretical results with the experimental data reported in Ref $\frac{82}{2}$. Our analysis at the level of the optical conductivity clearly confirms the pivotal role played by electronic correlations in the physics of $\mathrm{V}_{2} \mathrm{O}_{3}$ : the LDA results show a much stronger Drude peak (almost an order of magnitude stronger) when compared to the experiments. The inclusion of correlations via DMFT significantly improves the situation: Due to the proximity to the Mott-Hubbard MIT one observes a marked spectral weight shifts from the Drude peak to higher frequencies, which makes the overall agreement with experiment much better in the region up to $1.5 \mathrm{eV}$, where the experimental data are available.

From our analysis, moreover, another important aspect emerges: in the case of $\mathrm{V}_{2} \mathrm{O}_{3}$ the PA (adopted in previous calculations, e.g $\stackrel{37}{\underset{3}{2}}$ ) works satisfactorily well, at least in the low-energy $t_{2 g}$ subspace: The improvements due to the inclusion of the full optical matrix elements only leads to small changes in the optical spectra up to $2 \mathrm{eV}$ both in the LDA and LDA+DMFT results, as it can be expected on the basis of the discussion of Section IIB, considering the small (or vanishing) value of the crystal field splitting between the localized $t_{2 g}$ orbitals at the Fermi level.

\section{CONCLUSION}

We have developed a program package for calculating the optical conductivity on the basis of Wien2K, and make it available to the scientific community at www.wien $2 \mathrm{k}$.at/reg_user/unsupported/wien2wannier. Electronic correlations, e.g., from DMFT, or finite life times, e.g., from impurity scattering, can be included via a corresponding self energy for the Wannier orbitals. From this self-energy the Green function is calculated, which together with the full dipole matrix elements yields the optical conductivity, disregarding vertex corrections $\frac{18}{}$.

The main topic of the paper is a careful comparison between the dipole matrix element approach and the Peierls approximation, which is the de facto standard for lattice model calculations. We have considered two materials $\mathrm{SrVO}_{3}$ and $\mathrm{V}_{2} \mathrm{O}_{3}$ as testbeds. The low frequency part (below 2-3 eV) of the optical conductivity stems from $d-d$ transitions, at least for the two materials considered and many other transition metal oxides. This part is well captured by the Peierls approximation. One can understand this by the high degree of localization of the degenerate (or almost degenerate) Wannier $d$ orbitals: Below $1 \mathrm{eV}$, for both vanadates, it is also sufficient to include only the three $t_{2 g}$ bands out of the five $d$-orbitals. For the high frequency part (above $2-3 \mathrm{eV}$ ), on the other hand, not only the $d$ Hubbard bands are relevant but also $p-d$ transitions. This part of the spectrum is not well described by the Peierls approximation. Generalized Peierls approximation, while still approximate, also improves the description of $\mathrm{p}-\mathrm{d}$ transitions 10 at a computational cost comparable to the full dipole matrix calculation.

The comparison to experiment shows that LDA+DMFT with full dipole matrix elements gives a good description of the optical conductivity. In contrast, the Peierls approximation shows strong deviations at high frequencies. The same is true for the LDA optical conductivity even with the full dipole matrix elements. For instance, the LDA optical of $\mathrm{SrVO}_{3}$ conductivity particularly shows a too pronounced peak at $\sim 3.5 \mathrm{eV}$. This peak stems from $d-p$ transitions, and the DMFT correctly spreads the $d$ orbital spectral over a larger energy region: Hubbard side bands are formed and the electron-electron scattering smears out the $d$ bands.

The residual differences between LDA+DMFT and experimental infrared-spectra hence cannot be ascribed to the limitation of the Peierls approximation, but rather to effects beyond the LDA+DMFT scheme. For example, impurity scattering and the inclusion of non-local electronic correlations. The inclusion of the latter requires a considerable efforts of going beyond the standard LDA+DMFT scheme, e.g., by cluster extension of DMFT $\underline{83}$, dynamical vertex approximation $\underline{84}$ or duals fermion $\frac{85}{}$, which also necessarily requires a proper treatment of vertex corrections.

We thank P. Blaha, P. Hansmann, I.A. Nekrasov, S. Biermann, J. M. Tomczak and A. Pimenov for helpful discussions. JK, AT and KH acknowledge financial support from the Research Unit FOR 1346 of the Deusche Forschungsgemeinschaft and the Austrian Science Fund (FWF project ID I597-N16); PW was supported by the SFB ViCoM (FWF project ID F4103-N13) and GK 
W004. Calculations have been done on the Vienna Sci- entific Cluster (VSC).

1 D. N. Basov, R. D. Averitt, D. van der Marel, M. Dressel, and K. Haule, Rev. Mod. Phys. 83, 471 (2011).

2 G.D. Mahan, Many-Particle Physics, 2nd edition, Plenum Press, New York and London (1990)

3 For an introduction, see, e.g., R. Del Sole and R. Girlanda, Phys. Rev. B 48, 11789 (1993).

${ }^{4}$ R. O. Jones and O. Gunnarsson, Rev. Mod. Phys. 61689 (1989).

${ }^{5}$ L. Hedin, Phys. Rev. A 139796 (1965).

6 M. Rohlfing and S. G. Louie, Phys. Rev. B 62, 4927 (2000), S. Albrecht, L. Reining, R. Del Sole, and G. Onida, Phys. Rev. Lett. 80, 4510 (1998)

7 V. S. Oudovenko, G. Pálsson, S. Y. Savrasov, K. Haule, and G. Kotliar, Phys. Rev. B 70, 125112 (2004)

${ }^{8}$ K. Haule, V. Oudovenko, S. Y. Savrasov, and G. Kotliar, Phys. Rev. Lett. 94, 036401 (2005)

9 J. M. Tomczak and S. Biermann. J. Phys.: Condens. Matter 21,064209 (2009)

10 J. M. Tomczak and S. Biermann. Phys. Rev. B 80,085117 (2009)

11 J. M. Tomczak, K. Haule, G. Kotliar, Proc. Nat. Acad. Sci. USA 109, 3243 (2012)

12 J. M. Tomczak, "Spectral and optical properties of Correlated Material", PhD Thesis, Ecole Politechnique (2007).

13 W. Metzner and D. Vollhardt, Phys. Rev. Lett. 62324 (1989).

14 A. Georges and G. Kotliar, Phys. Rev. B 456479 (1992).

15 A. Georges, G. Kotliar, W. Krauth and M. Rozenberg, Rev. Mod. Phys. 6813 (1996).

16 G. Kotliar, S. Y. Savrasov and K. Haule and V. S. Oudovenko and O. Parcollet and C. A. Marianetti, Rev. Mod. Phys. 78, 865 (2006); K. Held, Adv. Phys. 56, 829 (2007).

17 K. Held. Advances in Physics 56,829-926 (2007)

18 When considering single band models in DMFT, the locality of all irreducible vertices ensures that all vertex correction in the optical conductivity exactly vanish 19,20 . While this may be not rigorously true for a (realistic) multiorbital case, it remains usually a good approximations ${ }^{12}$ when the DMFT assumptions are well fulfilled.

19 T. Pruschke, D. L. Cox and M. Jarrell, Phys. Rev. B 47 3553 (1993).

20 T. Pruschke, D. L. Cox and M. Jarrell, Europhys. Lett. 21 593 (1993).

21 N. Blümer, Ph.D. thesis, Universität Augsburg (2002).

22 P. Hansmann, M. W. Haverkort, A. Toschi, G. Sangiovanni, F. Rodolakis, J. P. Rueff, M. Marsi, and K. Held, Phys. Rev. B 85, 115136 (2012).

23 P. Blaha, K. Schwarz, P. Sorantin and S. Trickey. Computer Physics Communications 59,399 - 415 (1990)

24 The Wien2k calculations have been performed with 1000 k-points for $\mathrm{SrVO}_{3}$ and with 4000 k-points for $\mathrm{V}_{2} \mathrm{O}_{3}$. For the wien2wannier projections we used $1000 \mathrm{k}$-points for both materials.

25 G. H. Wannier. Phys. Rev. 52,191-197 (1937)

26 J. Kuneš, R. Arita, P. Wissgott, A. Toschi, H. Ikeda and K. Held. Computer Physics Communications 181,1888 1895 (2010)
27 F. Lechermann, A. Georges, A. Poteryaev, S. Biermann, M. Posternak, A. Yamasaki and O. K. Andersen. Phys. Rev. B 74,125120 (2006)

28 A. A. Mostofi, J. R. Yates, Y.-S. Lee, I. Souza, D. Vanderbilt and N. Marzari. Computer Physics Communications 178,685 - 699 (2008)

${ }^{29}$ M. Aichhorn, L. Pourovskii, V. Vildosola, M. Ferrero, O. Parcollet, T. Miyake, A. Georges and S. Biermann. Phys. Rev. B 80,085101 (2009)

30 J. E. Hirsch and R. M. Fye, Phys. Rev. Lett. 562521 (1986).

31 F. Aryasetiawan, M. Imada, A. Georges, G, Kotliar, S. Biermann and A. I. Lichtenstein, Phys. Rev. B 70, 195104 (2004).

32 B. Amadon, F. Lechermann, A. Georges, F. Jollet, T. O. Wehling and A. I. Lichtenstein. Phys. Rev. B 77,205112 (2008)

33 A. I. Poteryaev, J. M. Tomczak, S. Biermann, A. Georges, A. I. Lichtenstein, A. N. Rubtsov, T. Saha-Dasgupta and O. K. Andersen. Phys. Rev. B 76,085127 (2007)

${ }^{34}$ K. Held, G. Keller, V. Eyert, D. Vollhardt, and V. I. Anisimov, Phys. Rev. Lett. 86, 5345 (2001).

35 G. Keller, K. Held, V. Eyert, D. Vollhardt, and V. I. Anisimov, Phys. Rev. B 70, 205116 (2004).

${ }^{36}$ F. Rodolakis, P. Hansmann, J.-P. Rueff, A. Toschi, M. W. Haverkort, G. Sangiovanni, A. Tanaka, T. Saha-Dasgupta, O. K. Andersen, K. Held, M. Sikora, I. Alliot, J.-P. Itié, F. Baudelet, P. Wzietek, P. Metcalf and M. Marsi. Phys. Rev. Lett. 104,047401 (2010)

37 S. Lupi, L. Baldassarre, B. Mansart, A. Perucchi, A. Barinov, P. Dudin, E. Papalazarou, F. Rodolakis, J. -P. Rueff, J. -P. Iti Nature Communications 1, 105 (2010).

38 A Toschi, P Hansmann, G Sangiovanni, T Saha-Dasgupta, O K Andersen, K Held, Journal of Physics Conference Series 200, 012208 (2010).

39 I. A. Nekrasov, K. Held, G. Keller, D. E. Kondakov, T. Pruschke, M. Kollar, O. K. Andersen, V. I. Anisimov and D. Vollhardt. Phys. Rev. B 73,155112 (2006)

40 Sandvik, A. W.. Phys. Rev. B 57, 10287-10290 (1998)

41 C. Ambrosch-Draxl and J. O. Sofo. Computer Physics Communications 175,1 - 14 (2006)

42 P. Puschnig and C. Ambrosch-Draxl, Phys. Rev. B 66, 165105 (2002).

43 O. Peschel, I. Schnell and G. Czycholl, Eur. Phys. J. B 47 3, 369-378 (2005).

44 O. Gunnarsson, M. W. Haverkort, and G. Sangiovanni, Phys. Rev. B 82, 165125 (2010).

45 Peierls, R.. Zeitschrift fr Physik A Hadrons and Nuclei 80, 763-791 (1933)

46 R. Eguchi, T. Kiss, S. Tsuda, T. Shimojima, T. Mizokami, T. Yokoya, A. Chainani, S. Shin, I. H. Inoue, T. Togashi, S. Watanabe, C. Q. Zhang, C. T. Chen, M. Arita, K. Shimada, H. Namatame and M. Taniguchi. Phys. Rev. Lett. 96,076402 (2006)

47 A. Sekiyama, H. Fujiwara, S. Imada, S. Suga, H. Eisaki, S. I. Uchida, K. Takegahara, H. Harima, Y. Saitoh, I. A. Nekrasov, G. Keller, D. E. Kondakov, A. V. Kozhevnikov, T. Pruschke, K. Held, D. Vollhardt and V. I. Anisimov. 
Phys. Rev. Lett. 93,156402 (2004)

48 I. H. Inoue, I. Hase, Y. Aiura, A. Fujimori, K. Morikawa, T. Mizokawa, Y. Haruyama, T. Maruyama, and Y. Nishihara, Physica C 235-240, 1007 (1994);

49 T. Yoshida, M. Hashimoto, T. Takizawa, A. Fujimori, M. Kubota, K. Ono, and H. Eisaki Phys. Rev. B 82, 085119 (2010).

50 P. H. Dederichs, S. Blügel, R. Zeller and H. Akai, Phys. Rev. Lett. 532512 (1984); A. K. McMahan, R. M. Martin and S. Satpathy, Phys. Rev. B 386650 (1988); O. Gunnarsson, O. K. Andersen, O. Jepsen and J. Zaanen, Phys. Rev. B 391708 (1989).

51 I. A. Nekrasov, G. Keller, D. E. Kondakov, A. V. Kozhevnikov, T. Pruschke, K. Held, D. Vollhardt and V. I. Anisimov. Phys. Rev. B 72,155106 (2005)

52 E. Pavarini, S. Biermann, A. Poteryaev, A. I. Lichtenstein, A. Georges and O. K. Andersen. Phys. Rev. Lett. 92,176403 (2004)

53 A. Liebsch, Phys. Rev. Lett. 90, 096401 (2003);

54 Y. Z. Zhang and M. Imada, Phys. Rev. B 76, 045108 (2007)

${ }^{55}$ G. Trimarchi, I. Leonov, N. Binggeli, D. Korotin, V. I. Anisimov, arXiv:0802.4435.

56 P. Werner and A. J. Millis Phys. Rev. Lett. 104, 146401 (2010);

57 Takashi Miyake and F. Aryasetiawan, Phys. Rev. B 77, 085122 (2008);

58 V. I. Anisimov, D. E. Kondakov, A. V. Kozhevnikov, I. A. Nekrasov, Z. V. Pchelkina, J. W. Allen, S.-K. Mo, H.-D. Kim, P. Metcalf, S. Suga, and A. Sekiyama, G. Keller, I. Leonov, X. Ren, D. Vollhardt, Phys. Rev. B 71, 125119 (2005);

59 I.V. Solovyev, Phys. Rev. B 73, 155117 (2006);

60 E. Pavarini, A. Yamasaki, J. Nuss, O.K. Andersen, New J. phys. 7, 188 (2005);

61 F. Freimuth, Y. Mokrousov, D. Wortmann, S. Heinze, and S. Blügel. Phys. Rev. B 78, 035120 (2008).

${ }^{62}$ K. Byczuk, M. Kollar, K. Held, Y.-F. Yang, I. A. Nekrasov, Th. Pruschke and D. Vollhardt, Nature Phys. 168 (2007).

63 T. Yoshida, K. Tanaka, H. Yagi, A. Ino, H. Eisaki, A. Fujimori, and Z.-X. Shen, Phys. Rev. Lett. 95, 146404 (2005);

64 A. Toschi (unpublished).

${ }^{65}$ H. Makino, I. H. Inoue, M. J. Rozenberg, I. Hase, Y. Aiura and S. Onari. Phys. Rev. B 58,4384-4393 (1998)

66 R. J. O. Mossanek, M. Abbate, P. T. Fonseca, A. Fujimori, H. Eisaki, S. Uchida and Y. Tokura. Phys. Rev. B 80,195107 (2009)

67 R. J. O. Mossanek, M. Abbate, T. Yoshida, A. Fujimori, Y. Yoshida, N. Shirakawa, H. Eisaki, S. Kohno and F. C. Vicentin. Phys. Rev. B 78,075103 (2008)

68 E. Shiles, T.Sasaki, M. Inokuti, and D.Y. Smith, Phys. Rev. B 22, 1612 (1980).

69 D.Y. Smith, M. Inokuti, and W. Karstens Physics Essays 13, 465 (2000).

${ }^{70}$ H.J.A. Molegraaf, C. Presura, D. van der Marel, P. H. Kes, and M. Li, Science 295, 2239 (2002); A. F. Santander-Syro, R. P. S. M. Lobo, N. Bontemps, Z. Konstantinovic, Z. Z. Li, and H. Raffy, Phys. Rev. Lett. 88, 097005 (2002); A. Boris, N. N. Kovaleva, O. V. Dolgov, T. Holden, C. T. Lin, B. Keimer, and C. Bernhard, Science 304, 708 (2004); M. Ortolani, P. Calvani, and S. Lupi Phys. Rev. Lett. 94, 067002 (2005); F. Carbone et al., Phys. Rev. B 74, 064510 (2006); D. Nicoletti, O. Limaj, P. Calvani, G. Rohringer, A. Toschi, G. Sangiovanni, M. Capone, K. Held, S. Ono, Yoichi Ando, and S. Lupi, Phys. Rev. Lett. 105, 077002
(2010).

71 J. E. Hirsch, Science 295, 2226 (2002); A. Toschi, M. Capone, M. Ortolani, P. Calvani, S. Lupi, and C. Castellani, Phys. Rev. Lett. 95097002 (2005); L. Benfatto, S. G. Sharapov, N. Andrenacci, and H. Beck, Phys. Rev. B 71, 104511 (2005); A. Toschi, M. Capone, and C. Castellani Phys. Rev. B 72, 235118 (2005); J. P. Carbotte and E. Schachinger, J. Low Temp. Phys. 144, 61 (2006); F. Marsiglio, F. Carbone, A. B. Kuzmenko, and D. van der Marel, Phys. Rev. B 74, 174516 (2006); M. R. Norman, A. V. Chubukov, E. van Heumen, A. B. Kuzmenko, and D. van der Marel, Phys. Rev. B 76, 220509 (2007); A. Toschi and M. Capone, Phys. Rev. B 77, 014518 (2008).

72 C. Taranto, G. Sangiovanni, K. Held, M. Capone, A. Georges, A. Toschi, Phys. Rev. B 85085124 (2012).

73 A. Gössling, M. W. Haverkort, M. Benomar, Hua Wu, D. Senff, T. Möller, M. Braden, J. A. Mydosh, and M. Grüninger, Phys. Rev. B 77 , 035109 (2008).

74 D. B. McWhan, A. Menth, J. P. Remeika, W. F. Brinkman, and T. M. Rice, Phys. Rev. B 7, 1920 (1973).

75 L. Baldassarre, A. Perucchi, D. Nicoletti, A. Toschi, G. Sangiovanni, K. Held, M. Capone, M. Ortolani, L. Malavasi, M. Marsi, P. Metcalf, P. Postorino and S. Lupi. Phys. Rev. B 77,113107 (2008)

76 S.-K. Mo, J. D. Denlinger, H.-D. Kim, J.-H. Park, J. W. Allen, A. Sekiyama, A. Yamasaki, K. Kadono, S. Suga, Y. Saitoh, T. Muro, P. Metcalf, G. Keller, K. Held, V. Eyert, V. I. Anisimov, D. Vollhardt, Phys. Rev. Lett. 90, 186403 (2003).

77 J.-H. Park, L. H. Tjeng, A. Tanaka, J. W. Allen, C. T. Chen, P. Metcalf, J. M. Honig, F. M. F. de Groot, and G. A. Sawatzky, Phys. Rev. B 61, 11506 (2000).

78 M. J. Rozenberg, G. Kotliar, H. Kajueter, G. A. Thomas, D. H. Rapkine, J. M. Honig and P. Metcalf. Phys. Rev. Lett. 75,105-108 (1995)

79 The structure of the optical matrix elements, as well as (unpublished) LDA+DMFT calculations seem to indicate a relatively strong anisotropy between the optical conductivity measured perpendicularly or parallel to the $c$ axis (i.e., along the direction of the $\mathrm{V}-\mathrm{V}$ pairs in the conventional unit cell). This fact should be always kept in mind when analyzing experimental optical data, as even a small misalignment of the sample may alter considerably the predicted result.

80 A. I. Frenkel1, D. M. Pease, J. I. Budnick, P. Metcalf, E. A. Stern, P. Shanthakumar, and T. Huang, Phys. Rev. Lett. 97, 195502 (2006).

81 T. Saha-Dasgupta, O. K. Andersen, J. Nuss, A. I. Poteryaev, A. Georges, and A. I. Lichtenstein, arXiv:0907.2841.

82 M. J. Rozenberg, I. H. Inoue, H. Makino, F. Iga and Y. Nishihara. Phys. Rev. Lett. 76,4781-4784 (1996)

83 T.A. Maier, M. Jarrell, T. Pruschke, and M. H. Hettler, Rev. Mod. Phys. 77, 1027 (2005).

84 A. Toschi, A.A. Katanin, and K. Held, Phys. Rev. B 75, 045118 (2007).

85 A.N. Rubtsov, M.I. Katsnelson, and A.I. Lichtenstein, Phys. Rev. 77, 033101 (2008).

${ }^{86}$ D. Nicoletti, O. Limaj, P. Calvani, G. Rohringer, A. Toschi, G. Sangiovanni, M. Capone, K. Held, S. Ono, Yoichi Ando, and S. Lupi, Phys. Rev. Lett. 105, 077002 (2010).

87 I. H. Inoue, I. Hase, Y. Aiura, A. Fujimori, Y. Haruyama, T. Maruyama and Y. Nishihara. Phys. Rev. Lett. 74,2539$2542(1995)$ 
88 A. Fujimori, I. Hase, H. Namatame, Y. Fujishima, Y. Tokura, H. Eisaki, S. Uchida, K. Takegahara and F. M. F. de Groot. Phys. Rev. Lett. 69,1796-1799 (1992)

89 M. Imada, A. Fujimori and Y. Tokura. Rev. Mod. Phys. 70,1039-1263 (1998)

90 S. Itoh. Solid State Communications 88,525 - 527 (1993)

91 T. Maekawa, K. Kurosaki and S. Yamanaka. Journal of Alloys and Compounds 426,46 - 50 (2006)
92 Wannier, G. H.. Rev. Mod. Phys. 34, 645-655 (1962)

93 Maldague, P. F.. Phys. Rev. B 16, 2437-2446 (1977)

94 Scalapino, D. J., White, S. R. and Zhang, S. C.. Phys. Rev. Lett. 68, 2830-2833 (1992)

95 I. A. Nekrasov, G. Keller, D. E. Kondakov, A. V. Kozhevnikov, Th. Pruschke, K. Held, D. Vollhardt, and V. I. Anisimov, cond-mat/0211508; superceded by $\stackrel{47}{ }$. 